\title{
Analysis of Maximal-Ratio and Equal-Gain Diversity Systems for M-ary QAM on Generalized Fading Channels*
}

\author{
A. Annamalai ${ }^{1}$ and C. Tellambura ${ }^{2}$ \\ 1. Department of Electrical and Computer Engineering, University of Victoria, PO Box 3055 STN CSC, Victoria \\ BC V8W 3P6, Canada, Tel: +1-250-721-6043, Fax: +1-250-721-6048, E-mail: annamalai@ece.uvic.ca \\ 2. School of Computer Science and Software Engineering, Monash University, Clayton, Victoria 3168, Australia \\ Tel: +1-613-9905-3296, Fax: +1-613-9905-3402,E-mail: chintha@dgs.monash.edu.au
}

\begin{abstract}
New, simple yet very accurate closed-form expressions for calculating the symbol error probability (SER) of multilevel quadrature amplitude modulation (MQAM) in conjunction with L-fold antenna diversity on a Nakagami fading channel are presented. Both maximal-ratio (MRC) and equal-gain (EGC) predetection diversity combining techniques have been considered. The exact closed-form formula for MRC diversity system removes the limitations of previous studies in the literature which have been limited to Rayleigh fading. Moreover, an exact analysis of EGC for MQAM has not been reported previously despite its practical interest. The exact SER is expressed as a finite-range integral. Our unified expressions are sufficiently general to handle arbitrary fading parameters as well as dissimilar mean signal strengths across the diversity branches. The generality and computational efficiency of these new formulas render themselves as a powerful tool for SER analysis in different fading conditions.
\end{abstract}

\section{INTRODUCTION}

In recent years, MQAM modulation scheme has received much attention for facilitating high-rate data transmission over wireless links due to its inherent spectral efficiency. For instance, using 16-QAM modulation scheme coupled with pilot symbol assisted fading compensation technique and two antenna diversity reception, it is possible to facilitate $64 \mathrm{kbps}$ transmission with almost same channel spacing as that of present analog systems [1]. While M-ary QAM may be employed to increase the bandwidth efficiency, antenna diversity is usually needed to mitigate the effects of deep fades experienced on wireless links as well as to reduce the penaity in signal-to-noise ratio (SNR) due to co-channel interference. Besides, diversity methods play a crucial role in minimizing the transmit power requirements, particularly in the reverse link, because the battery capacity of handheld subscriber units is limited. Owing to the growing interest in MQAM modulation scheme, the importance of accurate and efficient computation of SER of MQAM with antenna diversity reception in different fading environments cannot be understated, and is of practical interest.

Previous work has included the following. The SER of MQAM in the additive white Gaussian noise (AWGN) is furnished in $[2,5-2-79]$ while the bit error rate (BER) of MQAM with two branch MRC diversity reception in Rayleigh fading is given in [3]. Subsequently, Kim et. al. [4] derived the BER of

\footnotetext{
* This work was supported in part by a Strategic Project Grant from the Natural Sciences and Engineering Research Council (NSERC) of Canada and in part by $\mathrm{BC}$ Tel Mobility Cellular.
}

MQAM with $L$-branch MRC diversity reception in Rayleigh fading using series expansion of the complementary error function, and the error rate expression is obtained in the form of infinite series. Lu et. al. [5] attained a new exact expression involving finite summations of hypergeometric functions for the SER with $L$-branch MRC diversity reception, also in Rayleigh fading channel. In [6], we derive a simple expression (involving finite summations of the MGF) for the SER of MQAM with MRC diversity over a Nakagami fading channel with arbitrary parameters by invoking a two-dimension GCQ formula. More recently, Alouini and Goldsmith [7] presented an expression for MQAM in terms of a finite-range integral by utilizing the alternative representation for the two-dimension Gaussian probability integral derived in [8] and the MGF method developed in [9] and [10]. By contrast, in this paper we enhance of our previous work by deriving several simple closed-form expressions for MQAM with $L$-fold MRC diversity on a Nakagami fading channel which require significantly fewer samples of the MGF than in [6]. Further, exact closed-form SER expressions are presented for three special cases of Nakagami fading.

In a related work on EGC, Altman and Sichak [11] have found an exact solution for the dual diversity system operating in a Rayleigh fading environment. For higher order of diversity, some approximations have been pursued in literature. This is due to the difficulty of finding a general closed-form expression for the resulting probability density function (PDF) of the sum of Nakagami distributed (or even Rayleigh distributed) random variables (RVs). For instance, Jakes [12] has made use of a small argument approximation for the PDF suggested by Schwartz et. al. [13]. Recently Beaulieu [14] has devised an infinite series technique to compute the PDF for the sum of independent Rayleigh RVs. Applying this technique, Beaulieu and Abu-Dayya [15] present a comprehensive study of EGC for coherent and differential binary signaling schemes by deriving a convergent infinite series for the complementary probability distribution of the SNR at the output of equal-gain combiner. Although their Fourier series approach may be extended to MQAM, but the analysis is rather involved and tedious. Since this method is computationally demanding, in this paper we developed an alternative, considerably simpler technique to evaluate the exact performance of EGC diversity systems. The new method requires only the knowledge of two Fourier transform identities and the application of the Parseval theorem. The finite-range integral can be estimated very accurately with only a few MGF samples using the GCQ formula. Our method is much simpler than that of suggested in [15] because the required characteristic function (CHF) can be found 
easily, and application of Parseval theorem circumvents the need to find the PDF of sum of Nakagami distributed RVs. The generality and computational efficiency of our new expressions render themselves as a powerful tool for SER analysis under a myriad of fading scenarios.

\section{SER OF MQAM WITH MRC DIVERSITY RECEIVER}

In the MQAM, a symbol is generated according to $\log _{2} M$ bits of source data, and each symbol in a quadrant has different SER. Among the various known signal constellations, rectangular QAM signal is the most frequently used in practice because [2]: (a) its signal constellation is easily generated as two PAM signals impressed on phase-quadrature carriers; (b) the task of signal demodulated can be performed without much difficulty; and (c) the average transmitted power required to attain a given minimum distance with rectangular QAM is only slightly higher than that of the best MQAM signal constellation. When $\log _{2} M$ is even (i.e., square QAM), the exact SER for MQAM in the AWGN channel is given by [2],

$$
P_{s}^{(E)}\left(\varepsilon \mid \gamma_{b}\right)=2 q \operatorname{erfc}\left(\sqrt{p \gamma_{b}}\right)-q^{2} \operatorname{erfc}^{2}\left(\sqrt{p \gamma_{b}}\right)
$$

where $q=1-1 / \sqrt{M}, p=1.5 \log _{2} M /(M-1)$, and $\gamma_{b}$ is the average received SNR per bit. On the other hand, when $\log _{2} M$ is odd, there is no equivalent $\sqrt{M}$-ary PAM system. In this case, the symbol error probability is tightly upper bounded by,

$$
P_{s}^{(O)}\left(\varepsilon \mid \gamma_{b}\right) \leq 2 \operatorname{erfc}\left(\sqrt{p \gamma_{b}}\right)-\operatorname{erfc}^{2}\left(\sqrt{p \gamma_{b}}\right)
$$

if the detector bases its decisions on the optimum distance metric (maximum likelihood criterion).

Next we outline several methods for computing the SER of MQAM with MRC diversity reception on a Nakagami fading environment. Each of this method is unique, interesting and novel in its own right. Hence, we are presenting them in the hope of stimulating further applications. It is also straight-forward to extend the analysis presented here for other fading distributions.

\section{A. Computation of SER using PDF of $\gamma_{b}$}

The average symbol error probabilities in a slow and flat Nakagami-fading channel may be derived by averaging the error rates for the AWGN channel over the PDF of the SNR at the output of the diversity combiner,

$$
P_{S}^{(E)}(\varepsilon)=\int_{0}^{\infty} P_{S}^{(E)}\left(\varepsilon \mid \gamma_{b}\right) p_{\gamma_{b}}\left(\gamma_{b}\right) d \gamma_{b} \equiv I_{1}-I_{2}
$$

where $\gamma_{b}=\left(E_{b} / N_{0}\right) \sum_{i=1}^{L} \alpha_{i}^{2}=\sum_{i=1}^{L} \gamma_{l}$ is the instantaneous SNR per bit with L-fold MRC diversity. The PDF of $\gamma_{b}$ is readily obtained by invoking the Fourier inversion theorem and noting that the PDF is real and symmetric about $t=0$ :

$$
p_{\gamma_{b}}\left(\gamma_{b}\right)=\frac{1}{\pi} \int_{0}^{\infty} \operatorname{Real}\left\{\phi_{\gamma_{b}}(t) \exp \left(-j t \gamma_{b}\right)\right\} d t
$$

where $\phi_{\gamma_{b}}(t)$ is CHF of $\gamma_{b}$ [2],

$$
\phi_{\gamma_{b}}(t)=\prod_{l=1}^{L}\left[\frac{m_{l}}{m_{l}-j t \bar{\gamma}_{l}}\right]^{m_{l}}
$$

with the assumption that the fading statistics across the $L$ antennas are uncorrelated (achieved through sufficient antenna separation). The parameter $m_{l}$ in (5) denotes the fading figure of the $l$ th diversity branch (i.e., antenna) and $\bar{\gamma}_{l}=\left(E_{b} / N_{0}\right) \Omega_{l}$ corresponds to the average received SNR of the $l$ th antenna.

In order to evaluate (3), let us first consider the following two Fourier transform identities:

$$
\begin{aligned}
G_{1}(\omega)=\int_{0}^{\infty} \exp (-j \omega t) \operatorname{erfc}(\sqrt{p t}) d t=\frac{1}{j \omega}\left[1-\frac{\sqrt{p}}{\sqrt{p+j \omega}}\right] \\
G_{2}(\omega)=\int_{0}^{\infty} \operatorname{erfc}^{2}(\sqrt{p x}) \exp (-j \omega x) d x \\
=\frac{1}{j \omega}\left[1-\frac{4}{\pi} \frac{\tan ^{-1}(\sqrt{1+j \omega / p})}{\sqrt{1+j \omega / p}}\right]
\end{aligned}
$$

Substituting (1) and (4) into (3), and then using the identities (6) and (7), we get an exact analytical expression for SER of MQAM in Nakagami fading channel with arbitrary parameters:

$$
P_{S}^{(\varepsilon)}(\varepsilon)=\frac{1}{\pi} \int_{0}^{\infty} \operatorname{Real}\left\{\phi_{\gamma_{b}}(t)\left[2 q G_{1}(t)-q^{2} G_{2}(t)\right]\right\} d t
$$

This one-dimension integral can be computed numerically (e.g., adaptive Simpson integration rule).

In some previous work (e.g., $[1,3,16]$ ), the authors have used an approximate SER formula for MQAM in fading channel by ignoring the second integral in (3) since $\operatorname{erfc}^{2}\left(\sqrt{p \gamma_{b}}\right)$ «erfc $\left(\sqrt{p \gamma_{b}}\right)$ as $\gamma_{b} \rightarrow \infty$. However, the discrepancy between the exact SER and that of calculated via the coarse approximation described above can be quite large even for moderate values of $\bar{\gamma}_{b}[6]$.

\section{B. Computation of SER using PDF of $\gamma_{b}$ and GCQ Formula}

By expressing (5) in the polar form and then making variable substitution $t^{2}+1 / 2=1 /(1+x),(8)$ may be re-stated as

$$
P_{S}^{(\varepsilon)}(\varepsilon)=\frac{q}{\pi} \int_{0}^{\infty} \frac{\psi(t)}{t \zeta(t)} d t=\frac{q}{\pi} \int_{-1}^{1} \frac{\xi(x)}{1-x^{2}} d x
$$

where $\zeta(t)=\prod_{l=1}^{L}\left[1+\left(t / \lambda_{l}\right)^{2}\right]^{m_{l} / 2}, \lambda_{l}=m_{l} / \bar{\gamma}_{l}$,

$$
\begin{gathered}
\psi(t)=\operatorname{Re}\left\{e^{j \theta(t)-\pi / 2}\left[2-q-\frac{2}{\sqrt{1+j t / p}}+\frac{4 q \tan ^{-1}(\sqrt{1+j t / p})}{\pi}\right]\right\}, \\
\xi(x)=\psi\left[\sqrt{\frac{1-x}{2(1+x)}}\right] / \zeta\left[\sqrt{\frac{1-x}{2(1+x)}}\right], \\
\text { and } \theta(t)=\sum_{l=1}^{L} m_{l} \tan ^{-1}\left(t / \lambda_{l}\right) .
\end{gathered}
$$

Now applying the GCQ formula of the first kind in (9), we get

$$
P_{S}^{(E)}(\varepsilon)=\frac{q}{n} \sum_{k=1}^{n} \xi\left[\frac{1}{\sqrt{2}} \tan \left(\frac{\pi(2 k-1)}{4 n}\right)\right] / \sin \left(\frac{\pi(2 k-1)}{2 n}\right)+R_{n}(10)
$$

Since the remainder term $R_{n}$ vanishes quickly as $n$ increases, $(10)$ is a rapidly converging series.

\section{Computation of SER using MGF of $\gamma_{b}$ and GCQ Formula}

Different from the conventional method for computing SER (i.e., direct evaluation of (3)), our third approach relies upon the knowledge of the MGF of $\gamma_{b}$, the use of an alternative exponential forms for one-dimension and two-dimension complementary error functions as well as the application of GCQ rule [20-22]. The MGF technique has been applied successfully in [6] but the second integral was evaluated with the aid of a two-dimension 
GCQ formula. In the following, we derive a much simpler closed-form SER formula for MQAM modulation scheme on Nakagami fading channels. The new expression reduces the number of MGF samples required to achieve a specified accuracy from $n(n+1)$ to $2 n$. This is mainly attributed to the alternative exponential representation of the two-dimension complementary error function:

$$
\operatorname{erfc}^{2}(\sqrt{x})=\frac{4}{\pi} \int_{0}^{\pi / 4} \exp \left[-x \csc ^{2}(\Theta)\right] d \Theta
$$

Since the MGF of $\gamma_{b}$ can be obtained from the CHF shown in (5) via relationship $\phi(s)=\phi_{Y_{b}}(j s)$, the SER of square QAM may be evaluated as

$$
P_{S}^{(\varepsilon)}(\varepsilon)=\frac{4 q}{\pi} \int_{0}^{\pi / 2} \phi\left(p \sec ^{2}(\Theta)\right) d \Theta-\frac{q^{2}}{\pi} \int_{0}^{\pi / 4} \phi\left(p \csc ^{2}(\Theta)\right) d \Theta
$$

or more conveniently using (13):

$$
\begin{aligned}
P_{S}^{(E)}(\varepsilon)= & \frac{2 q}{n} \sum_{i=1}^{n}\left\{\prod_{l=1}^{L}\left[1+\frac{p \sec ^{2}\left(\theta_{i}\right)}{\lambda_{l}}\right]^{-m_{l}}\right. \\
& \left.-\frac{q}{2} \prod_{l=1}^{L}\left[1+\frac{p \csc ^{2}\left(\theta_{i} / 2\right)}{\lambda_{l}}\right]^{-m_{l}}\right\}+R_{n}
\end{aligned}
$$

where $\theta_{i}=(2 i-1) \pi / 4 n$. The remainder term $R_{n}$ can be bounded using the results of Appendix $A$ in [21] and/or [22]. However, this is not necessary in practice, since one simply computes (13) for several increasing values of $n$, and stops when the result converges to a prescribed accuracy. Since (13) can approximate the true SER within any degree of accuracy, it can be viewed as an exact closed-form solution. Note the implications of (13): we are simply sampling the MGF at $n$ points. So as long as the MGF exists and computable, this method can work very effectively. In fact, its accuracy will be high if the high-order derivatives of the MGF vanishes rapidly.

\section{Computation of SER using Parseval's Theorem}

Our fourth technique for evaluating the SER of MQAM with MRC diversity relies on knowledge of two Fourier Transforms (FTs), the application of the Parseval's theorem and GCQ formula. By applying Parseval's theorem in (3), we get

$$
\begin{aligned}
P_{S}^{(E)}(\varepsilon) & =\frac{1}{2 \pi} \int_{-\infty}^{\infty} \operatorname{FT}\left[P_{s}^{(\varepsilon)}\left(\varepsilon \mid \gamma_{b}\right)\right] \phi_{\gamma_{b}}{ }^{*}(\omega) d \omega \\
& =\frac{1}{2 \pi} \int_{-\infty}^{\infty}\left[2 q G_{1}(\omega)-q^{2} G_{2}(\omega)\right] \phi_{\gamma_{b}}(\omega) d \omega
\end{aligned}
$$

where $\phi_{\gamma_{b}}(\omega), G_{1}(\omega)$ and $G_{2}(\omega)$ are defined in (5), (6) and (7), respectively. Notice that our method II-A and II-D are essentially the same. But the development of (14) is interesting because it lends itself into a unified-form of SER for MQAM with MRC diversity on arbitrary fading environments (not restricted to only Nakagami fading).

Now using variable substitution $\omega=\tan \theta$ in (14) and then applying GCQ formula, we get

$$
\begin{aligned}
P_{S}^{(\varepsilon)}(\varepsilon) & =\frac{1}{\pi} \int_{0}^{\pi / 2} \vartheta(\tan \theta) \sec ^{2} \theta d \theta \\
& =\frac{q}{2 n} \sum_{k=1}^{n} \vartheta\left[\tan \left(\frac{\pi(2 k-1)}{4 n}\right)\right] \sec ^{2}\left[\frac{\pi(2 k-1)}{4 n}\right]+R_{n}
\end{aligned}
$$

where $\vartheta(\omega)=\operatorname{Real}\left\{\left[2 G_{1}(\omega)-q G_{2}(\omega)\right] \phi_{r_{b}}(\omega)\right\}$.

\section{E. Exact Closed-Form Formulas for SER of MQAM with MRC}

In this subsection, we will present exact closed-form SER formulas of MQAM with MRC diversity for three special cases of Nakagami fading: (a) identical $\lambda_{l}=m_{l} / \bar{\gamma}_{i}$ across the cliversity branches and $\sum_{l} m_{l}$ is a positive integer; (b) fading severity index assumes an integer value and is common to all diversity branches, but $\lambda_{1}$ for $l=1, \ldots, L$ are dissimilar; and (c) distinct diversity branches and integer $m_{l}$ 's for $l=1, \ldots, L$.

Case (a): Let us assume $\lambda_{l}=\lambda$ for $l=1, \ldots, L$ and $\sum_{l} m_{l}=D$ is a positive integer. In this case, the random variable $y_{b}$ has a gamma PDF (obtained by inverting (5)),

$$
p_{\gamma_{b}}(\gamma)=\frac{\lambda^{D}}{(D-1) !} \gamma^{D-1} \exp (-\lambda \gamma)
$$

Then,

$$
\begin{aligned}
& I_{1}=4 q \int_{0}^{\infty} \frac{1}{2} \operatorname{erfc}(\sqrt{p \gamma}) p_{r_{b}}(\gamma) d \gamma \\
& =4 q\left[\frac{1}{2}\left(1-\sqrt{\frac{p}{\lambda+p}}\right)\right]^{D} \sum_{k=0}^{D-1}\left(\begin{array}{c}
D-1+k \\
k
\end{array}\right)\left[\frac{1}{2}\left(1+\sqrt{\frac{p}{\lambda+p}}\right)\right]^{k}
\end{aligned}
$$

using identity (14-4-15) in [2], and

$$
\begin{aligned}
I_{2} & =\frac{q^{2} \lambda^{D}}{(D-1) !} \int_{0}^{\infty} \gamma^{D-1} \exp (-\lambda \gamma) \operatorname{erfc}^{2}(\sqrt{p \gamma}) d \gamma \\
& =\frac{q^{2} \lambda^{D}(-1)^{D-1}}{(D-1) !}\left\{\left.\frac{d^{(D-1)}}{d s}\left[\frac{1}{s}-\frac{4}{\pi} \frac{\tan ^{-1}(\sqrt{1+s / p})}{s \sqrt{1+s / p}}\right]\right|_{s=\lambda}\right\}
\end{aligned}
$$

by exploiting the Fourier transform identity in (7). For small values of $D$, the $(D-1)$-th order differentiation in (13) can be computed by hand. For instance,

$$
\begin{gathered}
I_{2}=q^{2}\left[1-4 \mathfrak{I}+2 \kappa \lambda-\frac{2 \mathfrak{I} \lambda}{p+\lambda}\right] \text { for } D=2 ; \\
I_{2}=q^{2}\left[1-4 \mathfrak{I}+2 \kappa \lambda-\frac{2 \mathfrak{I} \lambda}{p+\lambda}+\frac{3 \kappa \lambda^{2}}{2(p+\lambda)}+\frac{\kappa \lambda^{2}}{2 p+\lambda}-\frac{3 \mathfrak{3} \lambda^{2}}{2(p+\lambda)^{2}}\right] \\
\text { for } D=3 ;
\end{gathered}
$$

where $\mathfrak{I}=\frac{\operatorname{atan}(\sqrt{1+\lambda / p})}{\pi \sqrt{1+\lambda / p}}$ and $\kappa=\frac{p}{\pi(p+\lambda)(2 p+i)}$.

If $D$ is large, then $I_{2}$ may be computed recursively (in terms of Gauss hypergeometric series):

$$
I_{2}=q^{2}\left[1-\frac{4}{\pi} \sum_{k=0}^{D-1} \frac{1}{(2 k+1)}\left(\frac{\lambda}{p}\right)_{2}^{k} F_{1}\left(\frac{1}{2}+k, 1+k ; \frac{3}{2}+k ;-1-\frac{\lambda}{p}\right)\right]
$$

Case (b): Let us assume that the fading severity index is common to all diversity branches and assumes an integer value. However, the average received SNR per branch $\bar{\gamma}_{i}$ may be different. In this case, we can write the PDF of $\gamma_{b}$ in the form,

$$
p_{\gamma_{b}}(\gamma)=\sum_{i=1}^{L} \sum_{k=1}^{m} A_{i k} \frac{m^{k} \gamma^{k-1}}{(k-1) ! \bar{\gamma}_{i}^{k}} \exp \left(\frac{-m \gamma}{\bar{\gamma}_{i}}\right)
$$

where

$$
A_{l k}=\left.\frac{(-m)^{m-k}}{(m-k) ! \bar{\gamma}_{l}^{m-k}} \frac{d^{m-k}}{d x^{m-k}}\left[\prod_{i=1, i \neq 1}^{L}\left(1-\frac{\bar{\gamma}_{i} x}{m}\right)^{-m}\right]\right|_{x=\frac{m}{\bar{\gamma}_{i}}}
$$


Then it is straight-forward to show that the SER may be expressed as,

$$
\begin{aligned}
& P_{S}^{(E)}(\varepsilon)=4 q \sum_{i=1}^{L} \sum_{k=1}^{m} A_{l k}\left[\frac{1}{2}\left(1-\mu_{l}\right)\right]^{k} \sum_{i=0}^{k-1}\left(\begin{array}{c}
k-1+i \\
i
\end{array}\right)\left[\frac{1}{2}\left(1+\mu_{l}\right)\right]^{i} \\
& -\left.q^{2} \sum_{l=1}^{L} \sum_{k=1}^{m} A_{l k} \frac{\lambda_{l}^{k}(-1)^{k-1}}{(k-1) !} \frac{d^{(k-1)}}{d s^{(k-1)}}\left[\frac{1}{s}-\frac{4}{\pi} \frac{\tan ^{-1}(\sqrt{1+s / p})}{s \sqrt{1+s / p}}\right]\right|_{s=\lambda_{l}}
\end{aligned}
$$

where $\mu_{i}=\sqrt{p \bar{\gamma}_{l} /\left(m+p \bar{\gamma}_{i}\right)}$. For the particular case of $m=1$, (22) reduces to the SER formula for square MQAM on Rayleigh fading channel derived in [5].

Case (c): If the diversity branches are distinct and $m_{l}$ 's assume integer values, we obtain, upon performing the inverse Laplace transform of (5),

$$
p_{\gamma_{b}}(\gamma)=\sum_{l=1}^{L} \sum_{k=1}^{m_{l}} \eta_{l}^{\left(m_{l}-k\right)} \frac{\gamma^{(k-1)} \lambda_{l}^{k} \exp \left(-\lambda_{l} \gamma\right)}{(k-1) !}
$$

where

$$
\eta_{l}^{\left(m_{l}-k\right)}=\left.\frac{1}{\left(m_{l}-k\right) ! \lambda_{l}^{k}}\left[\prod_{i=1}^{L} \lambda_{i}^{m_{i}}\right] \frac{d^{m_{l}-k}}{d s^{m_{l}-k}}\left[\prod_{i \neq l}^{L}\left(s+\lambda_{l}\right)^{-m_{l}}\right]\right|_{s=-\lambda_{t}}
$$

Then, the corresponding exact SER may be evaluated as

$$
\begin{aligned}
& P_{S}^{(E)}(\varepsilon)=4 q \sum_{l=1}^{L} \sum_{k=1}^{m_{l}} \eta_{l}^{\left(m_{l}-k\right)}\left[\frac{1}{2}\left(1-\sqrt{\frac{p}{\lambda_{l}+p}}\right)\right]^{k} \\
& \quad \times \sum_{i=0}^{k-1}\left(\begin{array}{c}
k-1+i \\
i
\end{array}\right)\left[\frac{1}{2}\left(1+\sqrt{\frac{p}{\lambda_{l}+p}}\right)\right]^{i}-q^{2} \sum_{l=1}^{L} \sum_{k=1}^{m} \eta_{l}^{\left(m_{l}-k\right)} \\
& \times\left.\frac{\lambda_{l}^{k}(-1)^{k-1}}{(k-1) !} \frac{d^{(k-1)}}{d s^{(k-1)}}\left[\frac{1}{s}-\frac{4 \tan ^{-1}(\sqrt{1+s / p})}{s \sqrt{1+s / p}}\right]\right|_{s=\lambda_{l}}
\end{aligned}
$$

To the best of the authors' knowledge, all the exact closed-form expressions for MQAM on Nakagami fading channel presented in this section are new.

\section{SER OF MQAM WITH EGC DIVERSITY RECEIVER}

The EGC is of considerable interest since it appears to offer comparable performance to the optimal MRC with much greater simplicity. In the following, we will derive an exact analytical expression for EGC for MQAM on Nakagami fading channel in terms of a finite-range integral. Our new approach circumvents the problem of computing the PDF of the sum of RVs (corresponding to the amplitude of the received signal) which is normally encountered in the analysis of EGC diversity receivers. Furthermore, the integral can approximated very precisely using the GCQ formula requiring the evaluation of a function at only a small number of points. This simple expression also handles arbitrary fading parameters as well as dissimilar mean signal strengths across the diversity branches.

In an equal gain combiner, the output of different diversity branches are first co-phased, equally weighted, and then summed to give the resultant output. The instantaneous SNR at the output of the EGC combiner is $\gamma_{b}=x^{2}$ where $x$ is defined as

$$
x=\sqrt{\frac{E_{b}}{L N_{0}}} \sum_{i=1}^{\iota} \alpha_{l}
$$

where $\alpha_{l}$ is a Nakagami RV with the statistical parameters $m_{l}$ and $\Omega_{l}$ as defined in Section II. Let $\bar{\gamma}_{k}=\Omega_{k}\left(E_{b} / N_{0}\right)$ denote the average SNR for the $k$-th branch, which is consistent with our definition for the MRC case. The CHF of $x$ (the sum of $L$ Nakagami RVs) in this case is simply the product of the individual CHFs, i.e.,

$$
\begin{aligned}
\phi_{x}(\omega)= & \prod_{k=1}^{L}\left[\Phi\left(m_{k}, \frac{1}{2} ; \frac{-\omega^{2}}{4 L \lambda_{k}}\right)\right. \\
& \left.+j \frac{\Gamma\left(m_{k}+1 / 2\right) \omega}{\Gamma\left(m_{k}\right) \sqrt{L \lambda_{k}}} \Phi\left(m_{k}+\frac{1}{2}, \frac{3}{2} ; \frac{-\omega^{2}}{4 L \lambda_{k}}\right)\right]
\end{aligned}
$$

The confluent hypergeometric function of the first kind $\Phi(a, b ; c)$ may be computed efficiently using a convergent series for small arguments and via a divergent expansion for large arguments [24].

From (1), the conditional error probability is

$$
P_{s}^{(E)}(\varepsilon \mid x)=2 q \operatorname{erfc}(\sqrt{p} x)-q^{2} \operatorname{erfc}^{2}(\sqrt{p} x)
$$

and we are interested in calculating its average over the Nakagami PDF, i.e.,

$$
P_{s}^{(E)}(\varepsilon)=\int_{0}^{\infty} P_{S}^{(E)}(\varepsilon \mid x) p_{x}(x) d x
$$

where $p_{x}(x)$ is the PDF of the sum of $L$ Nakagami random variables. It is difficult to invert (27) to get a closed-form expression for the PDF of $x$. Therefore, a Fourier series approach has previously been used. For our subsequent development, the following two Fourier transforms (FTs) are needed:

$$
\begin{aligned}
\Psi(\omega) & =\omega \int_{0}^{\infty} \operatorname{erfc}(x) \exp (j \omega x) d x \\
& =\frac{2}{\sqrt{\pi}} F\left(\frac{\omega}{2}\right)+j\left[1-\exp \left(\frac{-\omega^{2}}{4}\right)\right] \\
\varphi(\omega)= & \omega \int_{0}^{\infty} \operatorname{erfc}^{2}(x) \exp (j \omega x) d x \\
= & \frac{4}{\sqrt{\pi}} F\left(\frac{\omega}{2}\right)-\frac{4}{\sqrt{\pi}} F\left(\frac{\omega}{2 \sqrt{2}}\right) \exp \left(\frac{-\omega^{2}}{8}\right) \\
& +j\left[1-\exp \left(\frac{-\omega^{2}}{4}\right)-\frac{4}{\pi} F^{2}\left(\frac{\omega}{2 \sqrt{2}}\right)\right]
\end{aligned}
$$

where $F($.$) denotes the Dawson's integral,$

$$
F(x)=\exp \left(-x^{2}\right) \int_{0}^{x} \exp \left(t^{2}\right) d t=x \Phi\left(1, \frac{3}{2} ;-x^{2}\right)
$$

Now applying Parseval's theorem in (29), we get

$$
P_{s}^{(\varepsilon)}(\varepsilon)=\frac{1}{2 \pi} \int_{-\infty}^{\infty} \frac{1}{\omega}\left[2 q \Psi(\omega)-q^{2} \varphi(\omega)\right] \phi_{x}^{*}(\sqrt{p} \omega) d \omega
$$

Since the imaginary part of this integral is zero, we may re-write (33) in a more desirable form,

$$
P_{S}^{(E)}(\varepsilon)=\frac{q}{\pi} \int_{0}^{\infty} \frac{\beta(\omega)}{\omega} d \omega=\frac{2 q}{\pi} \int_{0}^{\pi / 2} \frac{\beta(\tan \theta)}{\sin (2 \theta)} d \theta
$$

where

$$
\beta(\omega)=\operatorname{Re}\left\{[2 \Psi(\omega)-q \varphi(\omega)] \phi_{x}^{*}(\sqrt{p} \omega)\right\}
$$

Note that (34) is an exact analytical solutions for MQAM with EGC diversity. Yet making another variable substitution $\omega^{2}+1 / 2=1 /(1+x)$ in (34) and then applying GCQ formula, we obtain a converging series representation for the EGC performance on Nakagami fading channel, 
$P_{s}^{(E)}(\varepsilon)=\frac{q}{n} \sum_{k=1}^{n} \beta\left[\frac{1}{\sqrt{2}} \tan \left(\frac{\pi(2 k-1)}{4 n}\right)\right] / \sin \left(\frac{\pi(2 k-1)}{2 n}\right)+R_{n}$

It is also interesting to note that (10) and (36) are in similar forms.

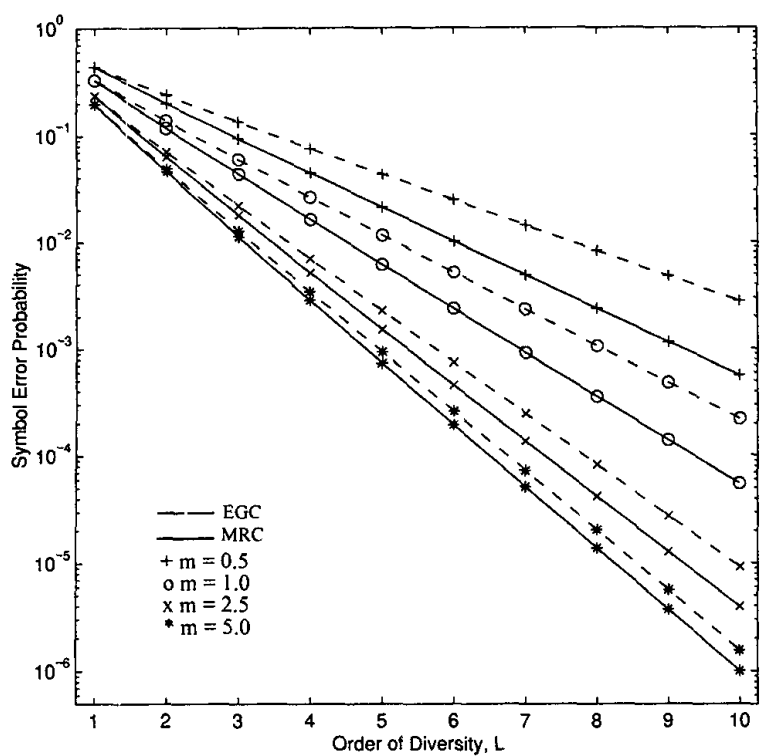

Fig 1. Symbol error probability versus order of diversity for 64-QAM with MRC and EGC diversity receivers. All the diversity branches are assumed to have identical fading statistics and the received SNR per branch is assumed to be $\bar{\gamma}_{c}=10 \mathrm{~dB}$.

\section{CONCLUSIONS}

New, simple yet very accurate symbol error probability expressions have been derived for coherent MQAM systems employing MRC and EGC antenna diversity in a Nakagami fading environment with an arbitrary fading severity index. The SER formula is exact for square QAM. A tight bound for the rectangular signal constellations was also presented. In particular, the closed-form formula based on GCQ can be easily programmed and evaluated efficiently. Our results are sufficiently general to allow for arbitrary fading parameters as well as dissimilar mean signal strengths across the diversity branches.

\section{ACKNOWLEDGMENT}

The authors are very grateful to Prof. Vijay K. Bhargava of University of Victoria for his continuous encouragement and guidance throughout this work.

\section{REFERENCES}

[1] S. Sampei, Applications of Digital Wireless Technologies to Global Wireless Communications, Prentice Hall, New Jersey, 1997.

[2] J. G. Proakis, Digital Communications, McGraw Hill, New York, Third Edition, 1995.

[3] T. Sunaga and S. Sampei, "Performance of multi-level QAM with post-detection maximal-ratio combining space diversity for digital land mobile radio communications," IEEE Trans. Vehic. Technology, Vol. 42, 99. 294-301, August 1993.
[4] C. J. Kim, Y. S. Kim, G. Y. Jung and H. J. Lee, "BER analysis of QAM with MRC space diversity in Rayleigh fading channel," Proc. PIMRC'95, pp. 482-485, September 1995.

[5] J. Lu, T. T. Tjhung and C. C. Chai, "Error probability perfornance of L-branch diversity reception of MQAM in Rayleigh fading," IEEE Trans. Commun., Vol, 46, pp. 179-181, February 1998.

[6] A. Annamalai, C. Tellambura and V. K. Bhargava, "Error Performance of M-ary QAM with MRC Diversity Reception in a Nakagami Fading Channel," IEEE International Symposium on Wireless Communications Digest, pp. 44, May 1998.

[7] M. Alouini and A. Goldsmith, "A Unified Approach for Calculating Error Rates of Linearly Modulated Signals over Generalized Fading Channels," Proc. ICC'98, Atlanta, pp. 459-464, June 1998.

[8] M. Simon and D. Divsalar, "Some New Twists to Problems Involving the Gaussian Probability Integral," IEEE Trans. Commun., Vol. 46, pp. 200-210, February 1998.

[9] C. Tellambura, A. J. Mueller and V. K. Bhargava, "BER and Outige Probability for the Land Mobile Satellite Channel with Maximal Ratio Combining," IEE Electronics Letters, April 1995.

[10] C. Tellambura, A. J. Mueller and V. K. Bhargava, "Analysis of M-ary Phase-Shift Keying with Diversity Reception for Land Mobile Satellite Channels," IEEE Trans. Vehicular Technology, Vol. 46, pp. 910-922, November 1997.

[11] F. J. Altman and W. Sichak, "A Simplified Diversity Communication System for Beyond the Horizon Links," IRE Trans. Commun. Systems, Vol. 4, pp. 50-55, March 1956.

[12] W. C. Jakes, Microwave Mobile Communications, New York: Wiley, 1974.

[13] M. Schwartz, W. R. Bennett and S. Stein, Communication Systems and Techniques, New York: McGraw-Hill, 1966.

[14] N. C. Beaulieu, "An Infinite Series for the Computation of the Complementary Probability Distribution Function of a Sum of Independent Random Variables and Its Application to the Sum of Rayleigh random Variables," IEEE Trans. Commun., Vol. 38, pp. 1463-1474, Sept. 1990.

[15] N. C. Beaulieu and A. Abu-Dayya, "Analysis of Equal gain Diversity on Nakagami Fading Channels," IEEE Trans. Commun., Vol. 39, pp. 225-234, February 1991

[16] W. T. Webb and L. Hanzo, Modern Quadrature Amplitude Modulation: Principles and Applications for Fixed and Wireless Channels, IEEE Press, New York, 1994.

[17] M. Nakagami, "The m-distribution - a general formula of inte ssity distribution of rapid fading," in Statistical Methods of Radio Wave Propagation, W. G. Hoffman Ed., Oxford, England: Pergamon, 1960, pp. 3-36.

[18] I. S. Gradshteyn and I. M. Ryzhik, Table of Integrals, Series and Products, Academic Press, 5th edition, 1995.

[19] M. Abramowitz and I. A. Stegun, Handbook of Mathematicai Functions, National Bureau of Standards, Applied Mathematics Series 5s, 1964.

[20] E. Biglieri, G. Caire, G. Taricco and J. Ventura-Traveset, "Sir ple method for evaluating error probabilities," IEE Electronics Letters, Vol. 32, February 1996, pp. 191-192.

[21] C. Tellambura, "Evaluation of the exact union bound for trellis coded modulations over fading channels," IEEE Trans. Communications, Vol. 44, pp. 1693-1699, December 1996.

[22] A. Annamalai, C. Tellambura and V. K. Bhargava, "Unified Analysis of MPSK and MDPSK with Diversity Reception in Different Fading Environments," IEE Electronics Letters, August 1998.

[23] A. Erdelyi, Higher Transcendental Functions, Vol. 1, McGraw-Hill, 1953.

[24] A. Annamalai, C. Tellambura and V. K. Bhargava, "Exact Evaluation of Maximal-Ratio and Equal-Gain Diversity Receivers for M-ary QAM on Nakagami Fading Channels," accepted for publication in the 'EEE Trans. on Communications. 